\title{
TRATAMENTO DE EFLUENTE ORIUNDO DA PRODUÇÃO DE QUEIJOS UTILIZANDO COAGULANTES NATURAIS
}

\author{
L. F. F. FARIA ${ }^{1}$, E. J. C. MORAES ${ }^{1}$, P. R. A. MENDES ${ }^{1}$ \\ ${ }^{1}$ Escola de Engenharia de Lorena/USP, Departamento de Engenharia Química \\ E-mail para figue@usp.br
}

\begin{abstract}
RESUMO - O uso de coagulantes naturais vem aumentando significativamente para o tratamento de efluentes devido às suas características biodegradáveis, além de gerar lodos menos poluentes que os coagulantes químicos. Em vista disso, no presente trabalho foram avaliadas as ações combinadas de três tipos de coagulantes naturais (quitosana, derivado de tanino e extrato de Moringa oleifera), diferentes valores de $\mathrm{pH}$ e formas de agitação visando a otimização da redução de matéria orgânica neste efluente lácteo, empregando a Metodologia da Superfície de Resposta. As variáveis resposta avaliadas foram: reduções de carbono orgânico total (COT), demanda química de oxigênio (DQO) e turbidez. Com este tratamento estatístico na condição ótima sugerida foram obtidas reduções de 19,1\% em COT, 13,6\% em DQO e 19,5\% em turbidez, usando 265 mg. $\mathrm{L}^{-1}$ de moringa, $430 \mathrm{mg} . \mathrm{L}^{-1}$ de tanino, agitação intermitente por $6 \mathrm{~h}$ e $\mathrm{pH}$ de 8,3 .
\end{abstract}

\section{INTRODUÇÃO}

Dentre os produtos derivados do leite, o queijo é o principal deles. A produção brasileira atingiu 722 mil de toneladas em 2013 gerando uma grande quantidade de efluentes e subprodutos, em destaque, o soro de queijo (Mapa, 2014). Para cada quilograma de queijo produzido são obtidos, em média, 10 litros de soro equivalendo a 7 milhões de toneladas de soro no Brasil por ano (Cuartas-Uribe et al., 2009). Este soro pode ser obtido a partir do leite por três processos: coagulação enzimática da caseína; precipitação ácida da caseína no ponto isoelétrico; e separação física das micelas de caseína por microfiltração, formando concentrado ou isolado proteico de micelas e proteínas do soro (Prazeres et al., 2012).

A disposição do excedente de soro de queijo não processado é um dos maiores inconvenientes encontrados pelas indústrias de laticínios. Janczukowicz et al. (2008) analisaram efluentes de laticínios oriundos de diferentes etapas da produção (estação de tratamento, seção de manteiga, de queijo e queijo cottage, recebimento de leite e sala dos equipamentos) e chegaram à conclusão de que o soro deve ser tratado separadamente dos outros efluentes, pois possui complexa biodegradação podendo sobrecarregar o sistema de tratamento. De acordo com Prazeres et al. (2012) o soro de queijo possui valores elevados de matéria orgânica, proporcionando variações na DQO de 50.000 a $102.000 \mathrm{mg} . \mathrm{L}^{-1}$ e na DBO de 27.000 a $60.000 \mathrm{mg} . \mathrm{L}^{-1}$, demonstrando uma capacidade de poluição 100 vezes maior que um efluente doméstico comum que, tipicamente, apresenta valores médios entre 250 a 800 mg. $L^{-1}$ de DQO e 110 a 500 mg. $L^{-1}$ de DBO (Castro, 2010). 
Assim, para o tratamento dos efluentes de soro de queijo devem ser consideradas três opções: a) Uso de tecnologias de recuperação de compostos com valor nutricional e funcional, como, por exemplo, proteínas e lactose; b) Emprego de tratamento biológico, com ou sem valorização dos subprodutos; c) Utilização de tratamentos físico-químicos para degradação de contaminantes, tais como matéria orgânica, turbidez e sólidos suspensos, também visando recuperação de produtos de alto valor agregado do soro.

Dentre os tratamentos convencionais disponíveis, destaca-se a coagulação/floculação por sua elevada eficiência na remoção de partículas coloidais e suspensas (minerais e orgânicas), que promovem turbidez e cor, não facilmente removidas por sedimentação (Pritchard et al., 2010). Primeiramente, pela adição do agente coagulante normalmente de carga positiva, promove-se a desestabilização das partículas por meio da neutralização das cargas eletrostáticas, fazendo com as mesmas se aglomerem gerando assim os flocos. Os flocos formados podem ser removidos em etapas posteriores por sedimentação, filtração direta ou flotação. Muitos fatores implicam diretamente nesta etapa como, por exemplo, o pH, o tipo e quantidade do coagulante, tempo de mistura e floculação do efluente.

As espécies utilizadas como coagulantes quanto a natureza química podem ser, por exemplo, sais de ferro III e alumínio, que apresentam elevada eficiência na remoção de materiais suspensos, no entanto, possuem desvantagens como a geração de grandes quantidades de lodo e de efluentes contendo metais, que podem colocar em risco a saúde humana. Em vista disso, vários estudos vêm sendo realizados utilizando coagulantes naturais com o intuito de substituir ou auxiliar os coagulantes convencionais. Estes coagulantes são biodegradáveis, poluem menos que os químicos, gerando assim lodos e efluentes de menor toxicidade (Cardoso et al., 2008; Pritchard et al., 2010).

Dentre os coagulantes naturais tem-se a quitosana que é extraída de fontes naturais, obtida a partir de animais marinhos como camarões, lagostas e caranguejos. Devido a sua alta densidade catiônica, tem grande afinidade por proteínas. Exemplo da ação efetiva da quitosana é apresentada por Sarkar et al. (2006) que compararam a ação de coagulantes químicos (sais de ferro e de alumínio), poliméricos (policloreto de alumínio) e naturais (ácido algínico, carbóxi-metil celulose sódica, quitosana) em efluentes de laticínios, destacando o efeito de baixas concentrações de quitosana (10-50 mg. $\left.\mathrm{L}^{-1}\right)$ nas reduções de DQO e de sólidos dissolvidos, respectivamente, de $57 \%$ e $48 \%$. Os taninos são derivados vegetais retirados de cascas de árvores como Acacia mearsii de Wild, Shinopsis balansae e Pinus pinaster, sendo compostos polifenólicos solúveis em água, com massa molar variando de 500 a alguns milhares de Daltons. Para estes coagulantes têm sido relatados testes bem sucedidos na remoção de surfactantes, corantes, remediação de efluentes domésticos e na clarificação de águas turvas (Pelegrino, 2011). E por fim, têm-se as sementes de Moringa oleifera, as quais podem ser utilizadas em processos diretos de adsorção química ou na ação de extrato coagulante obtido da semente. O emprego de tecnologias simples, facilidade de operação e manuseio são vantagens relacionadas ao uso do extrato coagulante que é biodegradável. Esta extração pode ser realizada com água ou na presença de sais. Okuda e colaboradores (1999) obtiveram extratos a partir da extração aquosa e de diversos sais, relatando que quaisquer sais conseguiram extrair maior quantidade de proteína coagulante da semente. 
Em vista disso, o objetivo deste trabalho foi avaliar a ação dos coagulantes naturais no tratamento de efluente oriundo da produção de queijo, determinando as condições ótimas a partir de planejamento experimental.

\section{MATERIAIS E MÉTODOS}

O efluente foi gentilmente cedido pela empresa Danubio (Dan Vigor Indústria e Comércio de Laticínios Ltda.), situada na cidade de Cruzeiro/SP. Após a coleta do material ao mesmo foi adicionado um biocida $(0,10 \%)$ e conservado em câmara fria a $4^{\circ} \mathrm{C}$.

Como coagulantes naturais foram utilizados a quitosana (grau técnico, da AldrichSigma), derivado de tanino Biosugar (Acquaquímica S/A) e sementes de Moringa oleifera (gentilmente cedidas pela Universidade Federal de Sergipe e Universidade Estadual de Maringá). Os reagentes analíticos utilizados possuem grau P.A. (Merck, Vetec, Synth, Aldrich). Os efluentes bruto e coagulado/floculado foram analisados quanto à DQO, DBO, COT, sólidos, $\mathrm{pH}$, condutividade e turbidez conforme Standard Methods. A lactose foi determinada por HPLC de fase reversa.

Os experimentos foram realizados a temperatura ambiente e, a partir das condições experimentais, foram ajustados os valores de $\mathrm{pH}$ do efluente com soluções de $\mathrm{H}_{2} \mathrm{SO}_{4}$ 2,5 mol. $\mathrm{L}^{-1}$ e NaOH 6,5 mol.L $\mathrm{L}^{-1}$. A seguir, o efluente foi transferido para cubas de Jar Test com volumes úteis de 1 litro, sendo adicionadas em cada cuba as quantidades requeridas de coagulantes com homogeneização a $120 \mathrm{rpm}$ por 1 minuto. Posteriormente, o sistema foi submetido a diferentes condições de agitação por um período de 6 horas. Foram preparadas soluções estoque de quitosana $\left(20 \mathrm{~g} . \mathrm{L}^{-1}\right)$ e derivado de tanino $\left(20 \mathrm{~g} . \mathrm{L}^{-1}\right)$. O extrato de Moringa oleifera $\left(20 \mathrm{~g} . \mathrm{L}^{-1}\right)$ foi obtido a partir de metodologia utilizada por Madrona et al. (2010). Para otimização desta etapa foi utilizada a Metodologia da superfície de resposta (MSR), estudando a ação das concentrações de coagulantes naturais, $\mathrm{pH}$ e agitação sobre as reduções de COT, DQO e turbidez. O estudo foi iniciado partindo de planejamento exploratório do tipo fatorial fracionado $2^{5-1}$, onde os níveis são mostrados na Tabela 1. Sequencialmente, foi realizado um novo planejamento exploratório, caminho máximo de ascensão e o delineamento central rotacional (DCCR). Os resultados foram analisados usando o software Minitab ${ }^{\circledR}$.

Tabela 1 - Variáveis de controle e respectivos níveis do planejamento fatorial $2^{5-1}$.

\begin{tabular}{cccc}
\hline Variável de Controle & \multicolumn{3}{c}{ Níveis } \\
\cline { 2 - 4 } & Baixo $(-1)$ & Central $(0)$ & Alto $(+1)$ \\
\hline Quitosana $\left(\mathrm{mg} . \mathrm{L}^{-1}\right)$ & 0 & 50 & 100 \\
Tanino $\left(\mathrm{mg} . \mathrm{L}^{-1}\right)$ & 0 & 50 & 100 \\
Extrato de moringa $\left(\mathrm{mg} . \mathrm{L}^{-1}\right)$ & 0 & 50 & 100 \\
pH & 4,0 & 7,0 & 10,0 \\
Agitação $(10 \mathrm{rpm})$ & Sem & Intermitente* & Constante \\
\hline
\end{tabular}

*a cada hora de experimento, 15 minutos ligado e 45 minutos desligado.

\section{RESULTADOS E DISCUSSÃO}

Após coleta do efluente recém-gerado, amostras foram caracterizadas e os resultados são mostrados na Tabela 2. O restante foi encaminhado à conservação. De acordo com a DQO 
e COT, este resíduo apresenta elevados teores de matéria orgânica dissolvida, principalmente, devido à presença de lactose. A razão $\mathrm{DBO} / \mathrm{DQO}$ de $0,13 \pm 0,02$ mostra que este efluente é recalcitrante e causaria sobrecarga em sistemas de tratamento biológico.

\begin{tabular}{cc}
\multicolumn{2}{c}{ Tabela 2 - Caracterização do efluente bruto. } \\
\hline Parâmetros & Valores \\
\hline pH & $6,56 \pm 0,01$ \\
Condutividade $\left(\mathrm{mS} . \mathrm{cm}^{-1}\right)$ & $7,42 \pm 0,04$ \\
DQO $\left(\mathrm{g} . \mathrm{L}^{-1}\right)$ & $260 \pm 5$ \\
COT $\left(\mathrm{g} . \mathrm{L}^{-1}\right)$ & $97,6 \pm 0,4$ \\
DBO $\left(\mathrm{mg} . \mathrm{L}^{-1}\right)$ & $34,9 \pm 0,7$ \\
Turbidez $(\mathrm{NTU})$ & $35,3 \pm 0,1$ \\
Sólidos totais $\left(\mathrm{g} . \mathrm{L}^{-1}\right)$ & $222,3 \pm 4,5$ \\
Sólidos dissolvidos $\left(\mathrm{g} . \mathrm{L}^{-1}\right)$ & $213,2 \pm 4,6$ \\
Lactose $\left(\mathrm{g} . \mathrm{L}^{-1}\right)$ & $209,4 \pm 0,2$ \\
\hline
\end{tabular}

A partir do primeiro planejamento exploratório (fatorial fracionado $2^{5-1}$ ), foram realizados os experimentos e ao final de cada ensaio foram analisadas reduções de COT, DQO e turbidez, gerando assim a matriz experimental (Tabela 3).

Tabela 3 - Matriz experimental do planejamento fatorial $2^{5-1}$ e as respectivas reduções de COT, DQO e turbidez.

\begin{tabular}{|c|c|c|c|c|c|c|c|c|}
\hline \multirow{2}{*}{ Exp. } & \multirow{2}{*}{$\begin{array}{l}\text { Quitosana } \\
\left(\mathrm{mg} \cdot \mathrm{L}^{-1}\right)\end{array}$} & \multirow{2}{*}{$\begin{array}{l}\text { Tanino } \\
\left(\mathrm{mg} \cdot \mathrm{L}^{-1}\right)\end{array}$} & \multirow{2}{*}{$\begin{array}{l}\text { moringa } \\
\left(\mathrm{mg} \cdot \mathrm{L}^{-1}\right)\end{array}$} & \multirow{2}{*}{$\mathrm{pH}$} & \multirow{2}{*}{ Agitação } & \multicolumn{3}{|c|}{ Redução } \\
\hline & & & & & & $\operatorname{COT}(\%)$ & DQO (\%) & Turbidez(\%) \\
\hline 1 & 0 & 0 & 0 & 4 & Constante $^{(1)}$ & 16,5 & 0,65 & 85,4 \\
\hline 2 & 100 & 0 & 0 & 4 & Sem & $-8,21$ & $-4,59$ & 65,2 \\
\hline 3 & 0 & 100 & 0 & 4 & Sem & 3,99 & 2,00 & 69,4 \\
\hline 4 & 100 & 100 & 0 & 4 & Constante $^{(1)}$ & 19,3 & 0,40 & 84,4 \\
\hline 5 & 0 & 0 & 100 & 4 & Sem & $-1,00$ & $-3,09$ & 56,6 \\
\hline 6 & 100 & 0 & 100 & 4 & Constante $^{(1)}$ & $-4,31$ & 4,05 & 67,8 \\
\hline 7 & 0 & 100 & 100 & 4 & Constante $^{(1)}$ & 10,0 & 1,24 & 16,6 \\
\hline 8 & 100 & 100 & 100 & 4 & Sem & 2,79 & 8,59 & $-20,7$ \\
\hline 9 & 0 & 0 & 0 & 10 & Sem & $-5,75$ & 1,32 & 93,5 \\
\hline 10 & 100 & 0 & 0 & 10 & Constante $^{(1)}$ & $-4,10$ & $-2,81$ & 85,7 \\
\hline 11 & 0 & 100 & 0 & 10 & Constante $^{(1)}$ & $-7,30$ & 4,22 & 80,5 \\
\hline 12 & 100 & 100 & 0 & 10 & Sem & $-1,53$ & $-2,76$ & 86,1 \\
\hline 13 & 0 & 0 & 100 & 10 & Constante $^{(1)}$ & 6,20 & 7,53 & 71,2 \\
\hline 14 & 100 & 0 & 100 & 10 & Sem & 8,04 & 2,93 & 78,7 \\
\hline 15 & 0 & 100 & 100 & 10 & Sem & 3,22 & 1,69 & 90,3 \\
\hline 16 & 100 & 100 & 100 & 10 & Constante $^{(1)}$ & 10,9 & 3,41 & 89,7 \\
\hline 17 & 50 & 50 & 50 & 7 & Intermitente $^{(2)}$ & 20,1 & 6,49 & 56,6 \\
\hline 18 & 50 & 50 & 50 & 7 & Intermitente $^{(2)}$ & 21,4 & 5,49 & 57,1 \\
\hline 19 & 50 & 50 & 50 & 7 & Intermitente $^{(2)}$ & 20,6 & 4,29 & 57,1 \\
\hline
\end{tabular}

${ }^{(1)}$ Velocidade de agitação: $10 \mathrm{rpm}$; ${ }^{(2)}$ a cada hora de experimento, 15 minutos ligado e 45 minutos desligado.

Como resultados de destaque observa-se que os experimentos 8 e 9 apresentaram as maiores reduções de DQO $(8,59 \%)$ e de turbidez $(93,5 \%)$ respectivamente. Para o COT, as maiores reduções foram alcançadas nos pontos centrais. Estes resultados foram submetidos à análise estatística através do teste "t" de Student e demonstraram que dentre os coagulantes estudados, o tanino e o extrato de moringa foram os mais significativos apresentando valores de $\mathrm{t}$ calculados iguais a 12,96 e 8,75 respectivamente, onde t crítico igual a 4,3 com $95 \%$ de 
confiança, portanto, a quitosana foi excluída dos planejamentos posteriores $(t=-1,13)$. A agitação e o pH também foram estatisticamente significativos de acordo com a mesma análise estatística, sendo o valor de $\mathrm{pH}$ igual a 10, aquele que possibilitou as maiores reduções de turbidez. Para a agitação, a condição intermitente foi a que proporcionou os melhores resultados de redução de COT, devido ao fato de esta modalidade possibilitar as colisões de intensidade moderada entre os coágulos pré-formados devido à agitação lenta gerando os flocos de maior dimensão. Na sequência, intervalos de repouso promoveram a sedimentação deste flocos maiores. A repetição destes ciclos aumentou a eficiência do processo. Resultados similares foram obtidos por Faria e Fonseca (2012) quando avaliando a técnica de coagulação/floculação em hidrolisado de bagaço de cana. Então, a partir deste planejamento foi proposto um $1^{\circ}$ DCCR visando maior precisão das condições experimentais ótimas. Para isso foram estabelecidas novas faixas para as variáveis controle mais significativas, sendo as novas faixas para as concentrações dos coagulantes tanino e extrato de moringa de 30 a 70 mg. $\mathrm{L}^{-1}$ e os valores de $\mathrm{pH}$ de 6,5 a 10,0 . A condição de agitação foi única para todos os experimentos na condição intermitente. A matriz experimental deste DCCR com os respectivos resultados de redução das repostas são mostrados na Tabela 4.

Tabela 4 - Matriz experimental do $1^{\circ}$ DCCR e as respectivas reduções de COT, DQO e turbidez.

\begin{tabular}{|c|c|c|c|c|c|c|}
\hline \multirow{2}{*}{ Exp. } & \multirow{2}{*}{ Moringa $\left(\mathrm{mg} . \mathrm{L}^{-1}\right)$} & \multirow{2}{*}{ Tanino $\left(\mathrm{mg} . \mathrm{L}^{-1}\right)$} & \multirow{2}{*}{ pH } & \multicolumn{3}{|c|}{ Redução } \\
\hline & & & & $\operatorname{COT}(\%)$ & DQO $(\%)$ & Turbidez (\%) \\
\hline 1 & 30 & 30 & 7,2 & 1,87 & 11,9 & -222 \\
\hline 2 & 70 & 30 & 7,2 & 8,68 & 7,99 & -197 \\
\hline 3 & 30 & 70 & 7,2 & 3,93 & 7,90 & -210 \\
\hline 4 & 70 & 70 & 7,2 & 6,74 & 12,0 & -176 \\
\hline 5 & 30 & 30 & 9,3 & 13,8 & 9,24 & 77,2 \\
\hline 6 & 70 & 30 & 9,3 & 2,12 & 12,9 & 76,4 \\
\hline 7 & 30 & 70 & 9,3 & 6,24 & 1,98 & 86,5 \\
\hline 8 & 70 & 70 & 9,3 & 7,28 & 12,0 & 83,3 \\
\hline 9 & 38,1 & 50 & 8,3 & 0,99 & 11,0 & $-11,6$ \\
\hline 10 & 61,9 & 50 & 8,3 & 4,43 & 12,2 & $-0,95$ \\
\hline 11 & 50 & 38,1 & 8,3 & 10,3 & 11,7 & $-4,21$ \\
\hline 12 & 50 & 61,9 & 8,3 & 17,3 & 12,0 & $-0,24$ \\
\hline 13 & 50 & 50 & 6,5 & 2,85 & 8,27 & -206 \\
\hline 14 & 50 & 50 & 10,0 & 0,84 & 12,2 & 80,3 \\
\hline 15 & 50 & 50 & 8,3 & 3,32 & 13,1 & 26,6 \\
\hline 16 & 50 & 50 & 8,3 & 1,98 & 12,7 & 27,8 \\
\hline 17 & 50 & 50 & 8,3 & 2,00 & 12,0 & 26,3 \\
\hline
\end{tabular}

Este novo planejamento permitiu uma investigação mais precisa dos efeitos das variáveis controle para redução das respostas, por consequência, abrangendo um maior número de pontos experimentais. A partir dos resultados obtidos foi possível observar que os experimentos 12, 6 e 7 mostraram os melhores resultados para as reduções de COT $(17,3 \%)$, DQO $(12,9 \%)$ e de turbidez $(86,5 \%)$, respectivamente, visto que o planejamento exploratório indicou que os melhores resultados poderiam ser obtidos com redução de concentração dos coagulantes. A partir da análise estatística dos resultados, por meio do teste "t" e da análise de variância, constatou-se que das respostas estudadas, a redução de COT foi a que apresentou modelo matemático válido e significativo, conforme a Equação (1). 


$$
\begin{aligned}
& \operatorname{COT}(\%)=2,43+0,686 . \text { Tanino }+0,347 . \text { Moringa }+0,356 \cdot p H \\
& +0,0975 . \text { Moring } a^{2}+4,01 \cdot \text { Tanino }{ }^{2}-0,208 \cdot p H^{2} \\
& +1,09 . \text { Tanino. Moringa }-0,318 . \text { Tanino. } p H-2,54 . \text { Moringa. } p H
\end{aligned}
$$

Contudo as análises dos melhores resultados obtidos neste $1^{\circ}$ DCCR também indicaram que melhores resultado poderiam ser obtidos em níveis mais elevados dos coagulantes, motivando a busca de outra região de máximo mais expressiva, o que foi feito através da metodologia do caminho máximo de ascensão. Então, para obtenção de uma representação bidimensional da superfície modelada com as curvas de nível, partiu-se do valor de redução de COT média obtida nos pontos centrais $(2,43 \%)$ do $1^{\circ}$ DCCR. Assim, o caminho máximo de ascensão foi determinado algebricamente a partir dos coeficientes do modelo para os coagulantes, sendo estabelecidos passos $(\Delta)$ de $50 \mathrm{mg} \mathrm{L}^{-1}$ e de $103 \mathrm{mg} \mathrm{L}^{-1}$ para a moringa e o tanino, respectivamente. $\mathrm{O}$ valor de $\mathrm{pH}$ foi mantido fixo $(8,3)$, devido as maiores reduções de turbidez, COT e DQO. A matriz experimental do caminho máximo de ascensão e os

\begin{tabular}{|c|c|c|c|c|c|c|}
\hline \multirow[b]{2}{*}{ Passos } & & & \multicolumn{4}{|c|}{ Redução } \\
\hline & \multicolumn{2}{|c|}{ Acréscimo na variável original $\left(\mathrm{mg}^{-L^{-1}}\right)$} & COT (\%) & DQO (\%) & Turbidez (\%) & \\
\hline$\Delta$ & 50 & 103 & - & - & - & \\
\hline Origem & 50 & 50 & 2,37 & 11,1 & 26,9 & - \\
\hline Origem $+\mathbf{1 \Delta}$ & 100 & 153 & 2,17 & 10,1 & 12,2 & - \\
\hline Origem $+\mathbf{2} \Delta$ & 150 & 256 & 2,04 & 9,94 & 2,74 & - \\
\hline Origem $+\mathbf{3} \boldsymbol{\Delta}$ & 200 & 359 & 1,09 & 7,38 & $-28,7$ & - \\
\hline Origem $+\mathbf{4 \Delta}$ & 250 & 462 & 7,57 & 8,79 & $-43,3$ & - \\
\hline Origem $+\mathbf{5} \Delta$ & 300 & 565 & 17,7 & 12,0 & $-45,5$ & - \\
\hline Origem $+6 \Delta$ & 350 & 668 & 8,59 & 3,66 & $-49,4$ & \\
\hline
\end{tabular}
resultados destes experimentos são mostrados na Tabela 5.

Tabela 5 - Caminho máximo de ascensão para condição de otimização da etapa de coagulação/floculação.

Pode-se observar que as reduções de COT, inicialmente foram diminuindo, mas a partir do $4^{\circ}$ experimento começaram a aumentar, atingindo um valor máximo de $17,7 \%$, e posteriormente voltaram a diminuir. Um comportamento análogo foi obtido com a redução de DQO. No entanto, a redução de turbidez apresentou um desempenho antagônico diminuindo com o decorrer do caminho de máxima ascensão. Possivelmente este comportamento aconteceu devido às elevadas concentrações dos coagulantes usados, aos quais cominou um efeito residual de partículas que não sedimentam e que, portanto, se mantiveram em suspensão. Deste modo, foi proposto um $2^{\circ}$ DCCR nesta região, que apresentou os melhores rendimentos, próxima das condições do $5^{\circ}$ experimento (moringa de $300 \mathrm{mg} . \mathrm{L}^{-1}$ e tanino de $\left.565 \mathrm{mg} . \mathrm{L}^{-1}\right)$. Os novos níveis de moringa e de tanino foram de 250 350 mg. $\mathrm{L}^{-1}$ e de $430-700 \mathrm{mg} . \mathrm{L}^{-1}$, respectivamente, e o pH sendo mantido constante em 8,3.

Para este $2^{\circ}$ DCCR foram avaliadas apenas as variáveis resposta COT e DQO, considerando que a turbidez apresenta tendência oposta de otimização das demais respostas. A matriz experimental do planejamento com os respectivos resultados de redução de COT e DQO são mostrados na Tabela 6. 
Tabela 6 - Matriz experimental do $2^{\circ}$ DCCR e respectivas reduções de COT e DQO.

\begin{tabular}{ccccc}
\hline & & & \multicolumn{2}{c}{ Redução } \\
\cline { 3 - 5 } Exp. & Tanino $\left(\mathbf{m g . L}^{-\mathbf{1}}\right)$ & Moringa $\left(\mathbf{m g . L}^{-\mathbf{1}}\right)$ & COT $(\boldsymbol{\%})$ & DQO (\%) \\
\hline 1 & 470 & 265 & $\mathbf{1 8 , 4}$ & $\mathbf{1 2 , 3}$ \\
2 & 660 & 265 & 13,2 & 10,9 \\
3 & 470 & 335 & 11,1 & 11,5 \\
4 & 660 & 335 & 16,6 & 11,7 \\
5 & 430 & 300 & 13,5 & 11,4 \\
6 & 700 & 300 & 14,3 & 11,7 \\
7 & 565 & 250 & 17,2 & 11,2 \\
8 & 565 & 350 & 15,7 & 6,81 \\
9 & 565 & 300 & 16,8 & 9,78 \\
10 & 565 & 300 & 17,9 & 9,74 \\
11 & 565 & 300 & 15,7 & 9,82 \\
\hline
\end{tabular}

Pelos resultados obtidos foi possível observar que o experimento 1 apresentou as maiores reduções de COT $(18,4 \%)$ e DQO (12,3\%). Aprofundando a análise estatística deste $2^{\circ}$ DCCR, observou-se que a redução de COT apresentou modelo matemático válido e significativo, gerando a superfície de resposta e curvas de contorno apresentados na Figura 1.

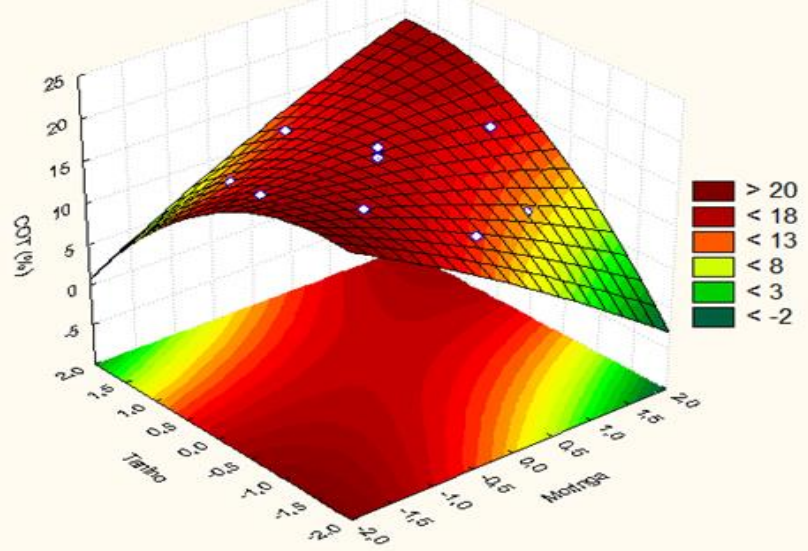

Figura 1 - Superfície de resposta e curvas de contorno para redução de COT em função da Moringa e Tanino.

Por fim, através do uso da função Desirability do software Minitab ${ }^{\circledR}$ e a partir dos modelos sugeridos para as respostas avaliadas foi determinada a condição ótima para as concentrações de tanino e moringa da etapa de coagulação/floculação sobre o efluente em estudo. Os valores encontrados foram de concentrações de $265 \mathrm{mg} . \mathrm{L}^{-1}$ de moringa e 430 mg. $\mathrm{L}^{-1}$ de tanino. A seguir foi realizada a validação experimental na condição otimizada, e como resultado foram obtidos valores de redução de COT, DQO e turbidez de 19,1\%, 13,6\% e $19,5 \%$, respectivamente.

\section{CONCLUSÃO}

A utilização da metodologia da superfície de resposta mostrou-se uma ferramenta eficiente para determinação das condições ótimas para a etapa de coagulação/floculação. 
Considerando ser este um efluente com uma carga orgânica elevada e de altos volumes gerados os valores de reduções obtidos, principalmente para COT e DQO podem representar um ganho considerável na redução do impacto ambiental deste efluente. Além do mais, pensando no uso combinado com outras tecnologias de purificação, estas reduções obtidas podem representar a viabilidade do uso de outras técnicas combinadas como, por exemplo, as Separações por Membranas. Além disso, a coagulação/floculação com diferentes coagulantes naturais mostrou-se uma alternativa eficiente ao uso de coagulantes químicos, gerando lodos também de menor impacto considerando a ausência de contaminantes metálicos oriundos do próprio coagulante.

\section{REFERÊNCIAS}

CARDOSO, K. C; BERGAMASCO, R; COSSICH, E.S; MORAES, C.K. Otimização dos tempos de mistura e decantação no processo de coagulação/floculação da água bruta por meio da Moringa oleifera . Acta Sci. Technol., v. 30, n.2, p. 193-198, 2008.

CASTRO, F.M.S. Estudo do comportamento hidrodinâmico de uma nova proposta de configuração interna para reator UASB. 102 p. 2010. Dissertação. Instituto de Pesquisas Hidráulicas, Universidade Federal do Rio Grande do Sul, 2010.

CUARTAS-URIBE, B.; ALCAINA-MIRANDA, M.I.; SORIANO-COSTA, E.; MENDOZA-ROCA, J.A.;; LORA-GARCIA, J. A study of the separation of lactose from whey ultrafiltration permeate using nanofiltration. Desalination, v. 241, p. 244-255, 2009.

FARIA, L.F.F.; FONSECA, C.R. Uso combinado de processos de separação visando a destoxificação do hidrolisado hemicelulósico. In: XIX COBEQ, 2012, Búzios. Anais do XIX COBEQ.

JANCZUKOWICZ, W.; ZIELINSKI, M.; DEBOWSKI, M. Biodegrability evaluation of dairy effluents originated in sections of dairy production. Bioresour.Technol., v. 99, p. 4199-4205, 2008.

MAPA (Ministério da Agricultura Pecuária e Abastecimento) - Sumário Executivo do Leite, Dezembro/2013. Disponível em: <http://www.agricultura.gov.br/politica-agricola/publicacoes/ > Acesso em: 04 abr 2014.

OKUDA, T.; BAES, A. U.; NISHIHJIMA, W.; OKADA, M. Improvement of the extraction method of coagulation active components from Moringa. Water Res., v. 33, p. 3373-3378, 1999.

PELEGRINO, E. C. F. Emprego de coagulante à base de tanino em sistema de pós-tratamento de efluente de reator UASB por flotação. 160 p. 2011. Dissertação. Escola de Engenharia de São Carlos, Universidade de São Paulo, 2011.

PRAZERES, A.R.; CARVALHO, F; RIVAS , J. Cheese whey management: A review. Sci. Total Environ., v. 445-446, p. 385-396, 2012.

PRITCHARD, M.; CRAVEN, T.; MKANDAWIRE, T.; EDMONDSON, A. S.; O’NEILL, J. G. A comparison between Moringa and chemicals coagulants in the purification of drinking water: Treatment, Disposal and Reuse. Phys. Chem. Earth, v. 35, p. 791-797, 2010.

SARKAR, B.; CHAKRABARTI, P. P.; VIJAYKUMAR, A.; KALE, V. Wastewater treatment dairy industries - possibility of reuse. Desalination, v. 195, p. 141-152, 2006. 